\title{
Aspectos Multidisciplinares dos Produtos do Processamento Aerogamaespectrométrico do Projeto São Timóteo - Uma Aplicação do Software Oasis Montaj'M 5.1
}

\author{
Esaú Francisco Sena Santos ${ }^{(1)}$, F. Fernando Lamego Simões Filho ${ }^{(2)}$, Jean Marie Flexor ${ }^{(3)}$ \\ ${ }^{(1)}$ Programa de Mestrado em Radioproteção e Dosimetria-IRD/CNEN/MCT, Bolsista Faperj; ${ }^{(2)}$ Serviço de Avaliação de \\ Impacto Ambiental, IRD/CNEN/MCT; ${ }^{(3)}$ Departamento de Geofísica, ON/MCT
}

\begin{abstract}
Resumo
O presente estudo trata da aplicação dos produtos do processamento aerogamaespectrométrico do Projeto São Timóteo, utilizando o software Oasis Montajт 5.1 da Geosoft $^{\circledR}$, visando demonstrar o potencial de sua aplicabilidade no campo da geologia e da proteção radiológica. As interpretações são realizadas analisando-se mapas de contorno gerados com os dados coletados nos canais do $\mathrm{K}, \mathrm{U}$ e Th, que são os radioelementos naturais utilizados na técnica.
\end{abstract}

\section{Introdução}

No ano de 1979 a GEOFOTO S.A., contratada pelas Empresas Nucleares Brasileiras - NUCLEBRAS, realizou um levantamento aerogeofísico na região sudoeste da Bahia, denominado de Projeto São Timóteo, onde foram reportadas medidas aerogamaespectrométricas com um detector de $\mathrm{Nal}(\mathrm{TI})$ sob uma altura média de $150 \mathrm{~m}$. Na época, pretendia-se identificar áreas que registrassem a ocorrência de anomalias radioativas com 0 intuito de prospectar urânio.

Este trabalho tem por finalidade aplicar os produtos do processamento (mapas de contorno) do levantamento aerogamaespectrométrico, do Projeto São Timóteo, no mapeamento de possíveis zonas preferenciais de recarga de água subterrânea e para determinar os níveis de radioatividade ambiental da área onde se encontram as anomalias uraníferas. Neste contexto, visou-se demonstrar o potencial multidisciplinar da aerogamaespectrometria. Tal técnica permite cobrir grandes áreas em curtos intervalos de tempo, a baixo custo e sem ofender o ambiente. Isto fornece uma visão macro das áreas de investigação, possibilitando que uma considerável quantidade de informações esteja, em um curto intervalo de tempo, disponíveis para otimização de diagnósticos sobre determinada área.

\section{Localização da área}

A região do levantamento do Projeto São Timóteo abrangeu uma área de $4.600 \mathrm{Km}^{2}$, localizada no sudoeste baiano. Os dados dessa pesquisa foram coletados das localidades de Caetité, São Timóteo e Lagoa Real, dentre outras. As coordenadas geográficas que determinam os limites da área prospectada são: - 13,5ํ№rte; -14,25 Sul; $-42,5^{\circ}$ Oeste; $-42^{\circ}$ Leste.

\section{Aquisição dos dados aerogamaespectrométricos}

Um levantamento aerogamaespectrométrico é composto por parâmetros que devem ser rigorosamente planejados e testados para garantir a qualidade dos dados adquiridos. A figura 1 mostra uma visualização dos parâmetros adotados no projeto São Timóteo. As medidas foram reportadas em quatro canais (potássio, urânio, tório e contagem total) em um detector da marca EXPLORANIUM DIGRS - 3001 de $\mathrm{Nal}(\mathrm{TI})$ com um volume de cristais de 1.017,87 polegadas cúbicas. As medidas foram tomadas em cps e posteriormente convertidas para $\mathrm{K}(\%)$, equivalente $\mathrm{U}$ (ppm) e equivalente Th (ppm). Considera-se que as séries do $U$ e do Th estejam em estado de equilíbrio secular e,

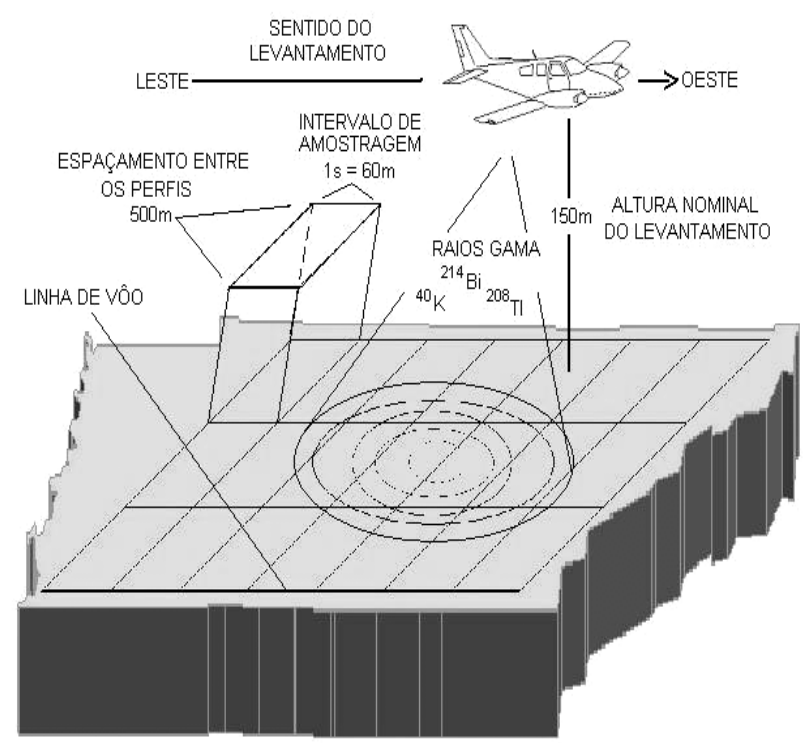

Figura 1 - Visualização dos parâmetros adotados no levantamento do Projeto São Timóteo

assim, determina-se os mesmos através de nuclídeos das suas séries de decaimento, respectivamente $0{ }^{214} \mathrm{Bi}$ $(1.76 \mathrm{MeV})$ e $0^{208} \mathrm{TI}(2.61 \mathrm{MeV})$, cujas energias apresentam espectro gama bem definido. Por outro lado, 0 ${ }^{40} \mathrm{~K}(1.46 \mathrm{MeV})$ é medido diretamente através de seu decaimento para $\circ \mathrm{Ar}^{40}$. Os dados foram corrigidos do efeito Compton, background cósmico e para altura nominal de vôo.

Processamento dos dados aerogamaespectrométricos

O software Oasis Montaj ${ }^{\mathrm{TM}} 5.1$ da Geosoft $^{\circledR}$ foi utilizado para 0 processamento dos dados aerogamaespectrométricos. Para construção dos mapas de contorno foi necessário realizar a transposição dos dados obtidos nas linhas de vôo para um formato digital em forma de uma malha regular quadrada (grid). O tamanho da célula adotado foi em torno de $1 / 4$ do espaçamento entre as linhas de vôo do levantamento conforme sugerido por (Vasconcellos et al, 1994). O algoritmo de interpolação foi executado utilizando-se a ferramenta BI-DIRECTIONAL GRIDDING, apropriada para levantamentos com linhas de 


\section{Aspec. Mult. dos Produtos do Proc. Aerogama. do Projeto São Timóteo}

vôo paralelas. O processo de geração das malhas regulares, denominado gridding, foi acompanhado de dois princípios básicos: (1) efetuou-se uma interpolação linear, ao longo das linhas de vôo, de direção (E-W), nos pontos correspondentes ao cruzamento de cada linha de vôo com cada meridiano teórico UTM (N-S) do grid; (2) efetuou-se uma interpolação (N-S), segundo os pontos definidos pelos cruzamentos dos paralelos UTM (E-W) com os meridianos (N-S), utilizando uma função para definição de cada nó do grid. A representação matricial supõe que o espaço pode ser tratado como uma superfície plana, onde cada célula está associada a uma porção do terreno. A resolução do sistema é dada pela relação entre o tamanho da célula no mapa e a área por ela coberta no terreno.

\section{Caráter multidisciplinar da aerogamaespectrometria}

A radioatividade pode ser empregada na prospecção de minerais e em mapeamento geológico. No Brasil, somente a partir da década de 50 é que a aerogamaespectrometria começou a ser empregada com o objetivo de prospecção de urânio (Vasconcellos et al, 1994). No caso da radioproteção, o emprego desta técnica representa um aumento na eficácia do mapeamento do fallout e do cálculo da dose de radiação ambiental, que possibilita determinar com exatidão o background em áreas de radioatividade natural elevada. Vários autores vêm demonstrando as aplicações da aerogamaespectrometria que culminam nos produtos de processamento aqui estudados (IAEA,1991; ICRU, 1994 \& Minty, 1997). Além disso, é importante ressaltar que os levantamentos aéreos permitem que vastas áreas sejam pesquisadas em curtos intervalos de tempo, melhorando a estatística de amostragem e conseqüentemente a interpretação dos resultados obtidos.

\section{Resultados}

Segundo (Soares, 2001), a pesquisa gamaespectrométrica requer no mínimo quatro mapas de contorno principais (potássio, equivalente urânio, equivalente tório e contagem total) representados aqui pelas figuras $2,3,4$ e 8 . A estatística inerente aos dados registrados nestes mapas está explicita na tabela 1 . Os mapas relativos as razões U/K, U/Th e Th/K também foram obtidos e estão representados pelas figuras 5,6 e 7 respectivamente. Todos os mapas de contorno demonstrados neste trabalho cobrem toda área de estudo.

Os mapas de contorno individuais ( $\mathrm{K}, \mathrm{U}, \mathrm{Th})$ refletem a distribuição destes radioelementos no ambiente bem como permite a visualização das anomalias radiométricas associadas a cada formação litológica. O mapa de contagem total demonstra o somatório das medidas radiométricas devido à contribuição do $\mathrm{K}, \mathrm{U}$ e Th. Já os mapas das razões têm por objetivo realçar as diferentes feições despercebidas nos mapas individuais.

\section{Discussão e Conclusões}

Baseando-se na tabela 1, percebe-se que o K apresenta os maiores teores, seguido do Th e do U. Isso pode ser devido ao fato de que normalmente $\circ \mathrm{K}$ e 0 Th são encontrados em forma de elementos traços na natureza, enquanto que o K é visto de forma mais abrupta.

Os dados relativos ao canal do $U$ apresentaram menor dispersão em torno da média, conseqüentemente tendo o menor desvio padrão, demonstrando assim ter uma distribuição no seu domínio espacial mais homogênea.

O K demonstrou o maior desvio padrão quando comparado ao Th e U. Isto pode estar ocorrendo devido ao fato de que este elemento geralmente não apresenta relação com a geologia do ambiente segundo (Vasconcellos et al, 1994). Ainda, pode-se inferir que o $\mathrm{K}$ devido ao valor obtido para seu desvio padrão, possa estar associado a vários tipos de solo do ambiente.

Em relação aos mapas das razões, (figuras 5, 6 e 7), percebe-se que a relação $\mathrm{Th} / \mathrm{K}$ apresenta os maiores valores. O tório demonstra estar mais concentrado do que o urânio, em relação ao potássio, conforme mostram as figuras 5 e 7 . Isto pode estar ocorrendo devido ao comportamento geoquímico do urânio, que tem por características ser mais solúvel do que o tório. Este comportamento também fica claro quando se observa a razão entre esses dois elementos na figura 6. Segundo (Vasconcellos et al, 1994) a razão entre os radioelementos tende a ser mais variável nas rochas mais antigas, logo a razão $\mathrm{Th} / \mathrm{K}$, figura 7 , pode estar demonstrando que estes dois radioelementos estão associados às formações litológicas mais antigas do ambiente.

Numa avaliação preliminar conclui-se que relativo a dosimetria ambiental o elemento $\mathrm{K}$ demonstrou ser o mais relevante. Quanto às razões U/K, U/Th e Th/K, ainda se faz necessário um estudo da litologia do ambiente associada ao comportamento geoquímico do $\mathrm{K}, \mathrm{U}$ e do Th para que se possa inferir sobre as possíveis zonas de recarga de água subterrânea. Também, é fundamental a execução de uma "filtragem" no domínio da freqüência dos dados para que se tenha um produto mais favorável a interpretação, eliminando desta forma os ruídos.

\section{Agradecimentos}

A CPRM, pela concessão dos dados e permissão para utiliza-los no trabalho de dissertação de mestrado do primeiro autor que culminou na realização deste trabalho. A CPG do IRD e a Faperj, pelo apoio na realização deste trabalho.

\section{Referências}

IAEA, 1991. Airborne Gamma-Ray Spectrometer Surveying, Technical Reports Series 323, International Atomic Energy Agency, Vienna.

ICRU, 1994, Gamma-Ray Spectrometry in the Environment, Report 53, International Commission on Radiation Units and Measurements, Maryland, USA.

MINTY, B.R.S., 1997. Fundamentals of Airborne Gammaray Spectrometry. AGSO Journal of Australian Geology and Geophysics, vol. 17, n.2, p.39-50. 


\section{Esaú Francisco Sena Santos, F. Fernando Lamego Simões Filho \& Jean Marie Flexor}

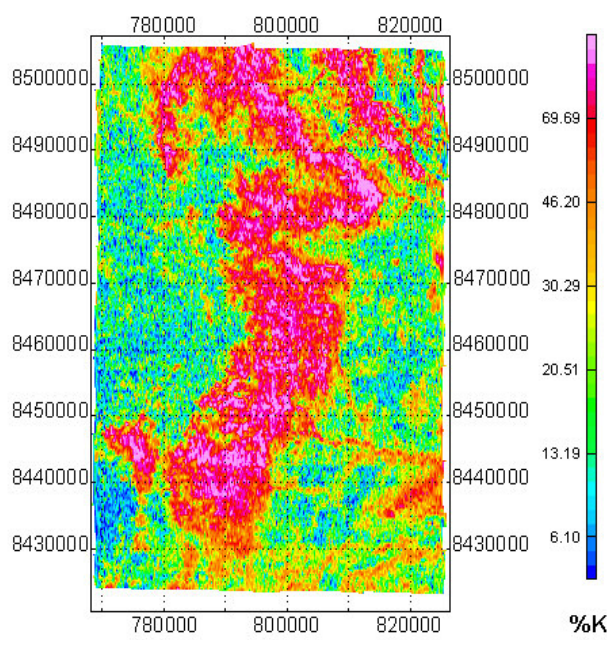

Figura 2 - Mapa de contorno do K

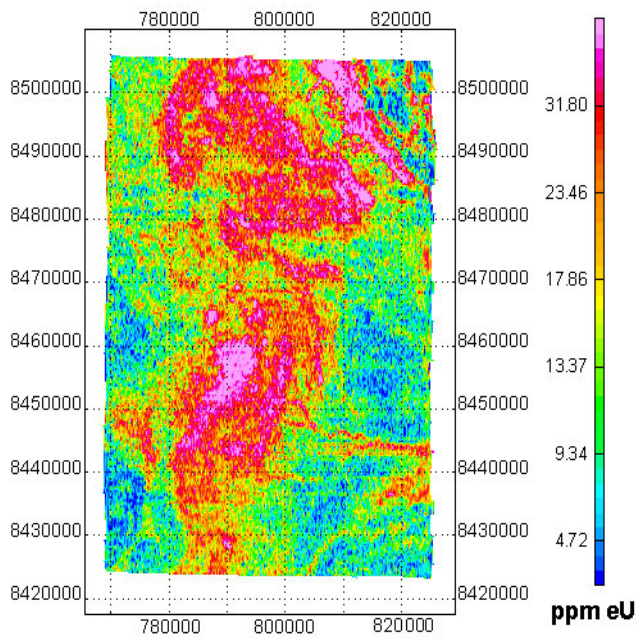

Figura 3 - Mapa de contorno do U

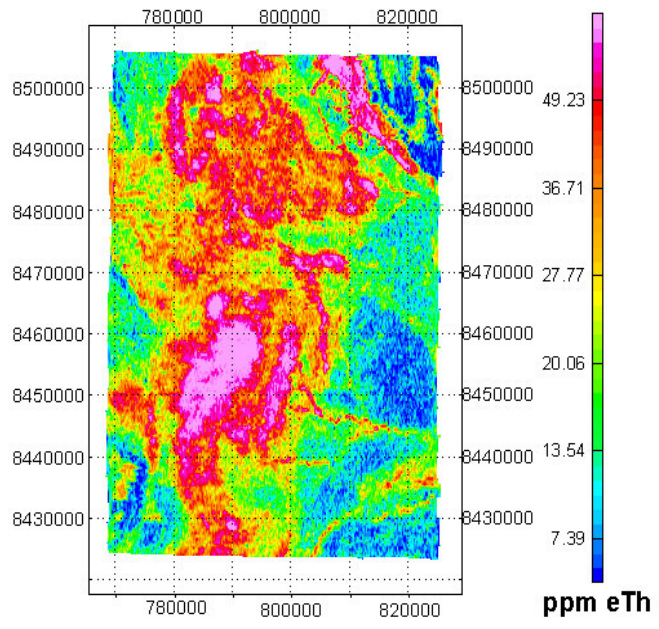

Figura 4 - Mapa de contorno do Th

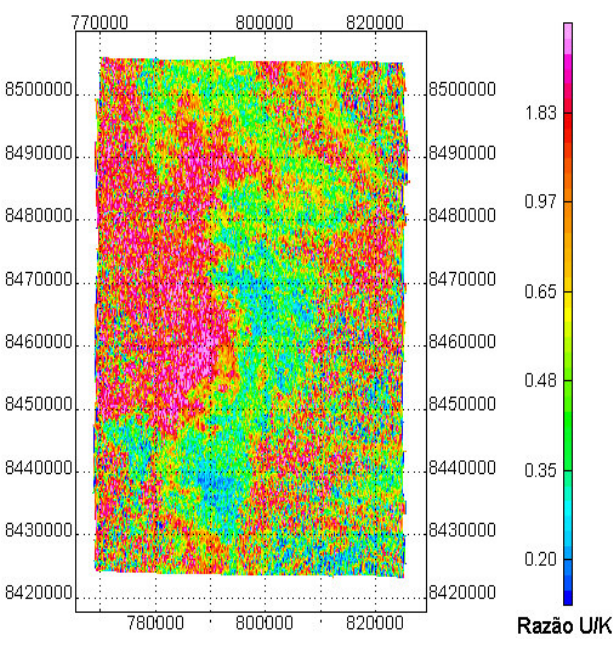

Figura 5 - Mapa de contorno da razão U/K

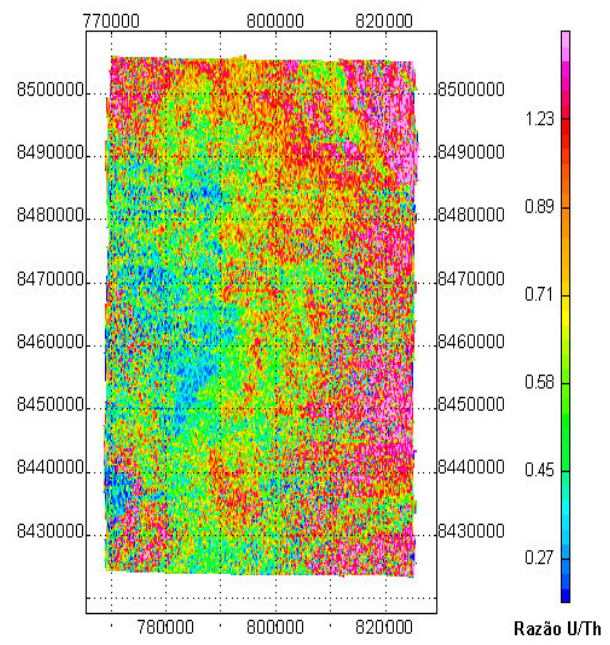

Figura 6 - Mapa de contorno da razão U/Th

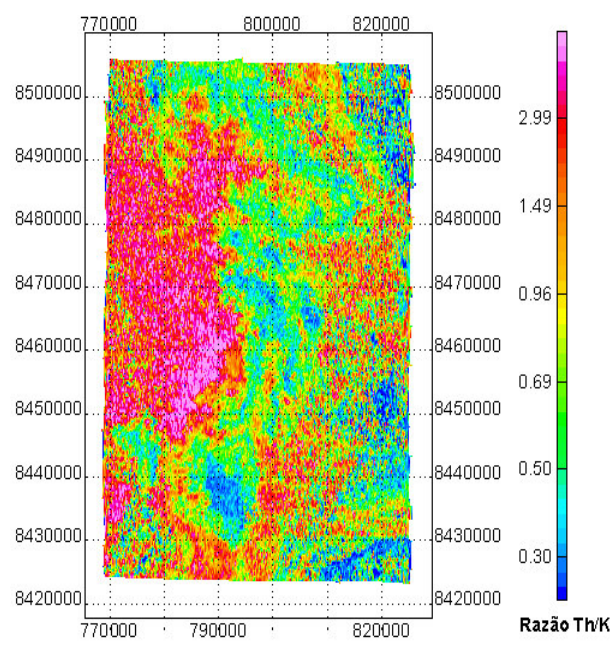

Figura 7 - Mapa de contorno da razão Th/K 
Aspec. Mult. dos Produtos do Proc. Aerogama. do Projeto São Timóteo

SO
AR
ES,
M.S
.,
200
1.
Pro
ces
sam
ento
e
Inte
rpre
taçã

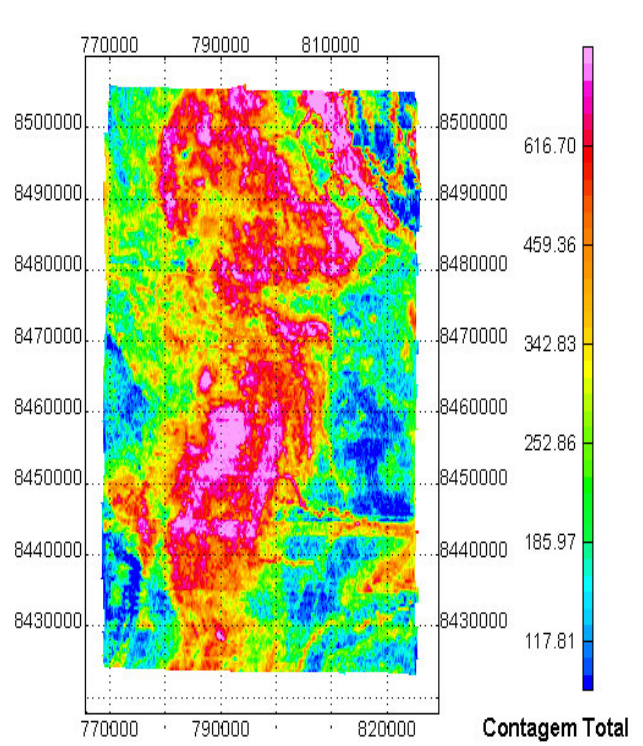

- de Dados Aerogeofísicos Magnetométricos e Gamaespectrométricos no Estudo do Controle das Mineralizações Auríferas na Região do Complexo Bossoroca, São Sepé, RS, Tese de Mestrado, PPGEM/UFRG, 161p.

VASCONCELLOS, R.M., METELO, M.J., MOTA, A.C.M., GOMES, R.D., 1994. Geofísica em Levantamentos Geológicos no Brasil, CPRM, Rio de Janeiro, 172p.

Figura 8 - Mapa de contorno de contagem total

\begin{tabular}{cccccc}
\hline CANAL & $\begin{array}{c}\text { NÚMERO DE } \\
\text { PONTOS } \\
\text { AMOSTRADOS }\end{array}$ & $\begin{array}{c}\text { VALOR MÍNIMO } \\
\text { OBSERVADO }\end{array}$ & $\begin{array}{c}\text { VALOR MÁXIMO } \\
\text { OBSERVADO }\end{array}$ & MÉDIA & $\begin{array}{c}\text { DESVIO } \\
\text { PADRÃO }\end{array}$ \\
\hline POTÁSSIO & 158725 & 0 & 260 & 37.15 & 30.11 \\
\hline URÁNIO & 158725 & 0 & 196 & 19.48 & 13.70 \\
\hline TÓRIO & 158725 & 0 & 205 & 30.63 & 21.84 \\
\hline $\begin{array}{c}\text { CONTAGEM } \\
\text { TOTAL }\end{array}$ & 158725 & 0 & 2076 & 379.64 & 235.64 \\
\hline
\end{tabular}

Tabela 1 - Estatística dos dados amostrados nos canais do K, U, Th e contagem total 